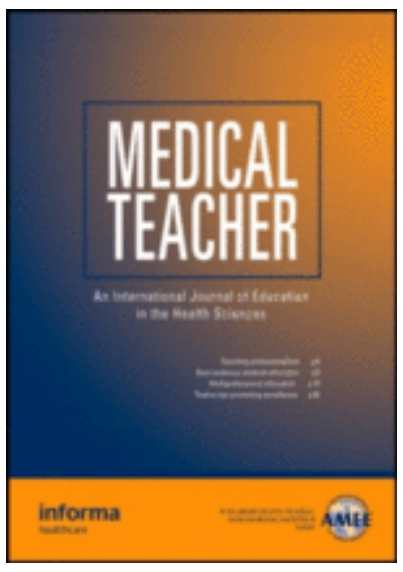

\title{
Using a self-regulated learning-enhanced video feedback educational intervention to improve junior doctor prescribing
}

\begin{tabular}{|c|c|}
\hline Journal: & Medical Teacher \\
\hline Manuscript ID & CMTE-2019-1478.R1 \\
\hline Manuscript Categories: & Articles \\
\hline $\begin{array}{r}\text { Date Submitted by the } \\
\text { Author: }\end{array}$ & $n / a$ \\
\hline Complete List of Authors: & $\begin{array}{l}\text { Patel, Rakesh; University of Nottingham School of Health Sciences, } \\
\text { Education Centre, School of Medicine; } \\
\text { Green, William; University of Leicester, } \\
\text { Shahzad, Muhammad; University of Leicester } \\
\text { Church, Helen; University of Nottingham School of Health Sciences, } \\
\text { Education Centre, School of Medicine } \\
\text { Sandars, John; Postgraduate Medical Institute }\end{array}$ \\
\hline Keywords: & $\begin{array}{l}\text { Simulation }<\text { Teaching \& Learning, Medical education research }< \\
\text { Management, Postgraduate }<\text { Phase of education }\end{array}$ \\
\hline
\end{tabular}

\section{SCHOLARONE Manuscripts}




\title{
Title: Using a self-regulated learning-enhanced video feedback educational intervention to improve junior doctor prescribing
}

\author{
Short title: SRL-enhanced video feedback to improve prescribing \\ Rakesh Patel ${ }^{1}$, William Green ${ }^{2, a}$, Muhammad Waseem Shahzad ${ }^{2}$, Helen Church ${ }^{1}$, John \\ Sandars ${ }^{3}$
}

a. Corresponding author:

Dr William Green

University of Leicester School of Business

University of Leicester, Leicester, LE1 7RH, UK.

Tel: 01162231233

w.green@le.ac.uk

Author affiliations:

1. School of Medicine, University of Nottingham, Nottingham, UK.

2. Innovation, Technology and Operations Division, University of Leicester School of Business, University of Leicester, Leicester, UK.

3. Health Research Institute, Faculty of Health, Social Care and Medicine, Edge Hill University Medical School, Ormskirk, UK. 


\title{
Title: Using a self-regulated learning-enhanced video feedback educational intervention to improve junior doctor prescribing
}

Short title: SRL-enhanced video feedback to improve prescribing

Authors and affiliations.

\begin{abstract}
Introduction

Medical school graduates in the UK consistently report feeling underprepared for the task of prescribing when embarking on practice. The effective application of self-regulated learning (SRL) approaches and feedback on complex tasks are associated with improved outcomes in practice-based clinical skills.
\end{abstract}

\begin{abstract}
$\operatorname{Aims}$
This study aimed to investigate the effectiveness of an educational intervention using SRL-enhanced video feedback for improving the prescribing competency of junior doctors.
\end{abstract}

\section{Methods}

A prospective cohort study was designed to compare intervention and control cohorts of junior doctors undertaking simulated clinical encounters at the beginning and end of their four-month rotation through renal medicine.

\section{Results}


The improvement in prescribing competency for the intervention cohort was significant $(\mathrm{p}<0.001)$ with large effect size $(d=1.42)$. Self-efficacy improved in both cohorts with medium effect size (control cohort $\mathrm{p}=0.026$, $d=0.64$; intervention cohort $\mathrm{p}=0.083, d=0.55$ ). Goal setting and selfmonitoring skills improved in the intervention cohort only with medium effect size $(\mathrm{p}=0.096, d=0.53)$.

\section{Conclusions}

SRL-enhanced video feedback is effective for improving prescribing competency and developing SRL processes such as goal setting and selfmonitoring skills in simulated clinical encounters. Further research is required to evaluate transferability to other clinical sub-speciality contexts and investigate the effectiveness of the intervention for improving prescribing in non-simulated settings.

Keywords: Deliberate Practice, Foundation Training, Junior Doctors, Patient Safety, Prescribing, Video-Enhanced Feedback 


\section{Introduction}

Prescribing medication is a key task for all doctors in practice (Charlton 2007).

Individuals need to know how to initiate, monitor, continue and modify medication.

This knowledge requires a thorough understanding of clinical pharmacology as well as the judgement and the ability to prescribe appropriately in practice (Kamarudin et al. 2013). Compared to other core clinical skills, such as venipuncture, developed across undergraduate education, prescribing requires competence in writing prescriptions, and effective diagnostic decision-making skills across different sub-specialty contexts. All these competencies need to be skillfully coordinated in order to perform safely in practice. This coordination of thinking whilst doing a task such as prescribing is known as metacognitive awareness. The first component of metacognitive awareness involves the ability of individuals to 'think about their thinking' whilst on task. The second component requires individuals to constantly 'regulate their thinking' by engaging in activities that improve the learning of skills needed to successfully complete the task (Schunk 2008). The extent to which medical students and graduates develop effective metacognitive awareness prior to graduation is unknown.

A number of studies consistently demonstrate medical students and new graduates feel underprepared for the task of prescribing before entering practice (Illing et al. 2013; Morrow et al. 2012; Wall et al. 2006). Specifically, the challenge individuals report back relate to the breadth of knowledge (ranging from pharmacological through to practical) required to prescribe competently, and the thinking needed to coordinate the various skills (ranging from self-monitoring to avoid error, through to interpersonal when working alongside pharmacists and nurses) required to prescribe safely (Illing et al. 2013). There are a number of reported educational interventions for developing prescribing skills (Ross and Loke 2009), developed in response to reported challenges 
in prescribing in junior doctors (Dornan et al. 2009; Patel et al. 2015); however, many interventions lack quality or evidence of effectiveness for improving the competency of new prescribers (Brennan and Mattick 2013; Kamarudin et al. 2013). The reasons are multifactorial. Firstly, a single intervention alone will not be able to develop the vast knowledge of drugs required to prescribe competently nor provide the clinical experience required to prescribe confidently across different sub-specialty contexts. Secondly, many interventions give greater emphasis to the technical aspects of prescribing ('what' and 'how' to write a prescription), rather than the metacognitive awareness required to know 'when' and 'why' to prescribe safely in practice. Likewise, metacognitive awareness is necessary in order to manage the various distractions when prescribing, with few interventions integrating human factors training as part of the education (Donisi et al. 2019).

Self-regulated learning (SRL), a socio-cognitive theory which emphasises the importance of metacognitive awareness and skills for effective performance (Zimmerman 1989), offers a new approach for informing the development of effective educational interventions for prescribing. SRL also provides a framework for the instructional design of teaching interventions, but also assessing the diagnostic decision-making components related to prescribing (Daniel et al. 2019), as well as guiding feedback approaches to those who also struggle on task (Durning et al. 2011). The application of SRL has been demonstrated across multiple areas of performance, including academic (Zimmerman 1990), sport (Kirschenbaum 1984), nursing practice (Kuiper and Pesut 2004) and undergraduate medical education (Heikkilä and Lonka 2006). SRL is a dynamic and cyclical process characterised by three sequential interrelated phases: forethought, performance and self-reflection (Zimmerman 1990). Forethought processes, such as self-efficacy beliefs, goal setting and strategic planning, 
precede any performance to motivate and direct individuals to choose the most appropriate techniques for completing the performance task. During the performance phase, self-monitoring ensures that chosen techniques remain appropriate to successfully completing the task. In the self-reflection phase following performance, individuals identify their beliefs about success or failure in performing the task, which are then adapted to achieve success in performing future tasks.

The effectiveness of SRL for improving complex cognitive skills, such as prescribing, remains relatively unexplored in authentic or real clinical environments. Feedback about SRL processes (SRL-enhanced feedback) is an essential component for giving effective feedback in general (Hattie and Timperley 2007). However, there is often little attention given to providing these aspects when providing feedback on prescribing in practice (Reynolds et al. 2016). More often than not, feedback given to junior doctors is minimal or not recognised as feedback (Bertels et al. 2013). When formally given, feedback on performance typically involves acknowledging the extent to which task-specific components were correctly completed by the learner, accompanied by personal evaluations of the learner by the educator (Reynolds et al. 2016).

There is growing appreciation that engaging the learner in two-way dialogue is essential to giving effective feedback but also ensures that information is not adversely received by the learner (Archer 2010). Feedback in conversational form or dialogue can be considered difficult to deliver due to perceived time pressures or the reluctance of educators to engage in difficult discussions about performance (Boud and Molloy 2013; Bowen et al. 2017). The aim of this study was to investigate the effectiveness of SRLenhanced video feedback for improving prescribing by junior doctors, and examining the utility of the technology to trigger conversation and stimulate reflection on 
performance against this backdrop. The hypothesis was that SRL-enhanced video feedback would increase the prescribing competence of junior doctors by improving awareness of SRL, thereby encouraging greater and sustained use of SRL processes among junior doctors over time when undertaking complex workplace tasks such as prescribing.

\section{Methods}

\section{Design and sample}

A prospective cohort design to compare intervention and control cohorts was used to establish the extent to which SRL-enhanced video feedback improved prescribing. Each cohort participated in simulated clinical encounters at the beginning and end of their four-month renal rotation in series (see Figure 1). This study design was chosen to mitigate cross-cohort contamination, meaning the control cohort participated in the April to July rotation and the intervention cohort participated in the August to November rotation. Given that the annual change for junior doctors in the UK takes place every August, the control cohort of doctors in their first two years after training (Foundation Years) were 'more experienced' as they entered the placement, in comparison to those in the intervention cohort, only by virtue of their position on the rotation across their training programme. Nevertheless, none of the participants had experience of a renal medicine placement on entry to the study.

Only the intervention cohort received SRL-enhanced feedback (indicated in Figure 1) after their simulated clinical encounters, facilitated by video footage of their simulation. Participants in both cohorts received the usual organised education provided across the rotation by the hospital Trust. 
$<$ Figure 1 near here $>$

\section{Research site}

The study was undertaken at the University Hospitals of Leicester (UHL) NHS Trust, United Kingdom. Junior doctors on the East Midlands South Foundation Training and Core Medical Training Programmes (EMLETB 2017) rotating to the Department of Nephrology, Leicester General Hospital, UHL NHS Trust, were invited to participate in the study. All junior doctors had participated in mandatory simulation training covering the management of acute medical emergencies and advanced life support at least once as part of their usual Foundation or Core Medical Training Programmes. Simulated clinical encounters were hosted at the Clinical Skills Unit, Leicester Royal Infirmary, UHL.

\section{Simulated clinical encounters}

Simulated clinical encounters involved completing a ward round with four 'real' patients (i.e. not actors). These were organised over two days to maximise the opportunity for participants to attend without compromising clinical service delivery. The encounters were also purposively designed to avoid being associated with final year simulation assessments through high realism, mimicking real ward practice: scans were available on COWs (Computers on Wheels), and nurses, pharmacists and senior doctors were available for consultation. Each patient had an authentic clinical problem related to one of four areas within renal medicine: acute kidney injury, chronic kidney disease, dialysis or transplantation. Patients were expected to behave authentically and describe real symptoms; scenarios were written around the patient's own clinical history. 
Prior to starting their ward round, each participant received a short, written handover list about each of the four patients. Thereafter, participants reviewed each patient in turn, during which they completed history-taking, physical examination and developed a list of differential diagnoses or clinical problems. Alongside the documentation of these activities, participants also constructed patient management plans and adjusted drug or fluid charts as appropriate. Participants completed each consultation by explaining their management plan to the patient and negotiating a shared outcome. Each participant completed these tasks for a single patient within 30 minutes. At the end of the ward round, participants updated the original handover list. For both cohorts, clinical simulated encounters were video recorded to account for the Hawthorne effect. For the intervention cohort, videos were subsequently used to deliver their SRL-enhanced feedback.

Immediately after participating, both cohorts were thanked and a debrief interview conducted regarding their experience. Participants were offered a copy of their personal, simulation patient-doctor interaction video recording to facilitate self-reflection. The intervention cohort were invited to attend a personalised 30-minute structured SRLenhanced video feedback session. During each session, participants reviewed the videos of their own patient encounters alongside a medical educator. Discussion focused around interactions with the patient, clinical diagnostic decision-making, medicines prescribed, interaction with other health care professionals and included discussions regarding the participant's use of key SRL processes, including self-efficacy, goal setting, strategy planning, self-monitoring and making adaptive changes. 


\section{Data collection and analysis}

Two types of data were collected during the simulated clinical encounters at the start and end of the four-month rotation: self-regulation (SRL) and prescribing competency data.

SRL data

A paper-based, simulation context-specific SRL questionnaire based on a microanalysis protocol (Cleary and Sandars 2011) was adapted, with responses either free-text or ranked on a scale of $0-100$. The questionnaire, pre- and post-simulation, was completed by each participant (See Table 1).

$<$ Table 1 near here $>$

Qualitative responses were transformed into quantitative data using an a priori coding framework. Two medical educators (author X and author Y) individually and independently coded all responses before reaching interrater agreement. The interrater reliability (McHugh 2012) was 85.99\% at first review (65 discrepancies from 464 coding tasks). Each code was assigned a numerical value reflecting the level of selfregulatory behaviour demonstrated. For example, responses pertaining to the processes involved in achieving a task were assigned a higher value than those that focused solely on the outcome of the task. Each SRL question was assigned a maximum of two marks to achieve an even weighting within the questionnaire. Therefore, the codes for each open question (questions 2-6, 8, 9 and 11) had a maximum of two marks, and the scale response (questions 1, 7 and 10) divided by 50. The maximum SRL score was 22 across the 11 questions with two marks available for each. 
In addition to analysing the total SRL scores, two specific SRL processes were investigated: 'self-efficacy' and 'goal setting/self-monitoring'. They were analysed using data only from questions which specifically aligned with these skills (questions 1 , $3,4,5,6$ and 7 , respectively).

\section{Prescribing competency data}

Each simulated clinical encounter involved a different prescribing task in accordance with the General Medical Council's Good Practice Guide on prescribing and managing medicines and devices standards (GMC 2013). The clinical diagnostic decision-making aspects of the prescribing task were derived from the Royal College of Physicians Generic Note Keeping Standards (RCP 2015) and Ward Rounds in Medicine Principles for Best Practice (RCP 2012). The participants' prescriptions were scored using a scenario-specific checklist devised by a medical educator (author X) and the departmental pharmacist (author Y). For each candidate a mean competency score was calculated across their four patient encounters.

\section{Participant debrief interview data}

Immediately following the simulations, de-brief interviews were conducted. Questions focused on the participant perceptions of the simulations including i) the usefulness of the simulations in additional to their usual education ii) any anxiety provoked by participating in such an intervention (Sørensen et al. 2017) and iii) the utility of the SRL-enhanced video feedback. 


\section{Statistical analysis}

Paired samples t-tests or Wilcoxon signed rank tests were used to measure the changes in the means or medians of competency and SRL scores for normal and non-normal data, respectively (Swinscow and Campbell 1997). Pearson correlation was used to quantify the association between variables. In addition to p-values, the results also report effect sizes using Cohen's $d$ (Cohen 1992). Reporting effect sizes is encouraged in medical education research (Sullivan and Feinn 2012), as it provides a fuller picture of the results, particularly in cases where the sample sizes are small - the likelihood of rejecting the null hypothesis increases as the sample size increases (Cohen 1992). In this study, effect size shows the magnitude of differences in scores pre- and post-simulated clinical encounters.

\section{Ethics}

The study was undertaken as part of the University Hospitals of Leicester NHS Trust's clinical effectiveness programme (study reference number 6608E) and Health Education England's quality improvement and innovation initiative (study reference number LEI0085). The study did not require full NHS ethics approval.

\section{Results}

There were 18 participants in this study: 6 in the control cohort and 12 in the intervention cohort. Participant demographics are shown in Table 2.

$<$ Table 2 near here $>$ 


\section{Change in prescribing competence and SRL scores}

Table 3 presents the pre- and post-intervention prescribing competency and SRL scores (total score and individual SRL process scores) for both cohorts.

$<$ Table 3 near here $>$

The Intervention cohort demonstrated a significant $(\mathrm{p}<0.001)$ improvement in prescribing competency, with a large effect size $(d=1.42)$. The Control cohort demonstrated a smaller improvement in their prescribing competency.

Regarding SRL, total scores were not significant for either cohort. However, selfefficacy did significantly improve in the Control $(p=0.026)$, but not in the Intervention $(\mathrm{p}=0.083)$, cohort. Furthermore, the effect size for both the Control cohort and the Intervention cohort was medium $(d=0.64$ and $d=0.55)$.

Goal setting and self-monitoring skills improved in the Intervention cohort $(p=0.096)$ but decreased in the Control cohort $(p=0.246)$. The effect size was medium for both cohorts (Cohort 1-control $d=0.55$, Cohort 2-intervention $d=0.53$ ).

\section{Correlations between prescribing competency and SRL processes}

Further analyses were undertaken to establish any correlation between prescribing competency and each of the individual SRL processes (self-efficacy, and goal setting and self-monitoring). The scores for simulation 1 and simulation 2 are arranged in cross-tabulation (Table 4). There was a significant correlation between the simulation 2 self-efficacy and prescribing competency score $(r=0.591, p=0.043, n=12)$.

$<$ Table 4 near here $>$ 


\section{Perceptions of the simulations and feedback}

Thirty-six debrief interviews were conducted (two per participant) in total, lasting between 6 and 25 minutes per interview. Table 5 provides illustrative quotes from the interviews. All participants from both cohorts suggested the simulations were of value to their learning and that their peers should be given the opportunity to participate in the intervention. Participants confirmed experiencing anxiety before and during the simulations even though their engagement with the intervention was voluntary, and had no bearing in any formal summative assessment or evaluation of performance. Participants believed the fidelity of the simulations, specifically the use of real patients and scenarios enhanced their learning. Participants who received the intervention felt watching themselves perform back on video was particularly powerful for their personal learning and reflection. Furthermore, their learning from this experience was enhanced by the dialogue that ensured with a medical educator, even though the process also felt uncomfortable at times.

$<$ Table 5 near here $>$

\section{Discussion}

This research adds to the body of evidence describing educational interventions that improve the competence of new prescribers such as junior doctors. The study demonstrates the effectiveness of video-enhanced feedback focusing on key SRL processes observed when individuals undertake complex workplace-based tasks such as prescribing (see Table 3). The study suggests that video is an effective trigger for starting feedback conversations with junior doctors about goal setting and selfmonitoring, when undertaking difficult or challenging prescribing in simulated clinical encounters. Finally, the findings confirm that feedback intended to increase self- 
efficacy (i.e. praise alone) is insufficient for maintaining prescribing competence over time (see Table 4).

\section{Educational prescribing interventions}

Teaching generic prescribing skills and good practice principles as outlined by the WHO Good Prescribing Guide (de Vries et al. 1994) has traditionally formed the basis of many educational interventions (Ross and Loke 2009). In reality, multiple interventions are now used to improve prescribing by junior doctors, ranging from opportunistic feedback on prescribing in the workplace (Bertels et al. 2013), through to timetabled sessions organised by pharmacists as part of a formal training programme (McLellan et al. 2016). A growing number of prescribing interventions are multifaceted and include a combination of simulated clinical encounters: eLearning, workplacebased teaching, feedback and access to reference aids (Larose et al. 2017; Morgan et al. 2017; Reynolds et al. 2016). The challenge now for educators is to develop ways for mainstreaming the delivery of SRL-enhanced video feedback post-simulated clinical encounters for complex cognitive tasks such as prescribing. For simple skill-based tasks, the benefits of using video feedback are well-established (Farquharson et al. 2013; Hachambachari et al. 2017; Naik et al. 2018; Parker et al. 2019). In some cases, there is evidence that improvements in clinical skills development using video are equally effective (Phillips et al. 2017) if not more effective (Nesbitt et al. 2015) in comparison to feedback received directly from an expert.

That said, this study also demonstrated that the use of video for complex clinical skills needs further exploration, with benefits unlikely to be seen without feedback dialogue between educator and learner, given that prescribing skills are multifaceted and require a review of knowledge, understanding and skills development in practice-based settings 
(Parker et al. 2019). Whilst direct observation and feedback on performance in practice remains commonplace in healthcare professions education (Caldwell 2011; LaDonna et al. 2017), there remains a curious reaction to the prospect of digitally recording performance on video in a training context (Bing et al. 2018). Learners actively seek direct observation and microanalysis of performance in domains such as sport in order to improve in the future (Middlemas and Harwood 2018). However, within medical education there is growing recognition of a curious phenomenon whereby learners instinctively want to be watched and receive granular feedback, however may also feel reluctant to take up opportunities for close observation when they perceive scrutiny of performance may prevent progression in training (Farquharson et al. 2013; LindonMorris and Laidlaw 2014; Nilsen and Baerheim, 2015). The challenge for medical educators is to reconcile such a tension given the significant benefits of close observation of complex skills such as prescribing, and reflection triggered by watching performance again alongside an educator.

\section{SRL processes}

The use of SRL-enhanced feedback is growing in undergraduate medical education (Artino et al. 2011; Dunphy et al. 2010; Heikkilä and Lonka 2006; Tanner 2012). This research adds to the growing case for giving SRL-enhanced feedback in postgraduate healthcare education contexts following complex diagnostic decision-making tasks such as prescribing. Encouraging greater metacognitive awareness as part of the debrief following high-fidelity simulations is commonplace (Duffy et al. 2015). However, SRL processes which underpin metacognition are not always included as part of the debrief when prescribing errors are discussed in simulated or actual practice. The majority of prescription errors result from individuals 'not knowing enough' about the use of 
medicines in different clinical contexts rather than insufficient knowledge about the patients or drug in and of themselves (Dean et al. 2002). This intervention was designed so that emphasis was given to supporting individuals to learn how to think through complex prescribing tasks, rather than replicate more teaching about particular drugs or clinical problems in the debrief following simulated clinical encounters. Furthermore, the debrief also focused on exploring the specific SRL processes of goal setting, selfmonitoring and self-efficacy demonstrated by junior doctors when prescribing for patients presenting with uncommon or challenging pathophysiological states such as renal failure (Bates et al. 1995; Bobb et al. 2004; Dean et al. 2002).

\section{Goal setting and self-monitoring}

Appropriate goal setting is also important so that individuals 'set off on the right track' when prescribing medication for patients with complex diseases; and self-monitoring is important so that individuals learn to identify 'when they are going off track' rather than avoid the task of prescribing, which is known to cause patients harm (Franklin et al. 2011). By using simulated clinical encounters to investigate SRL processes and prescribing behaviours, educators can better examine individual monitoring judgements and explicitly examine the factors that led individuals to formulate their judgements (de Bruin et al. 2017), especially when associated with a prescribing error. Effective selfmonitoring among experts on reasoning tasks is associated with individuals slowing down as they come up against difficulty, and even prompting a change in behaviour such as seeking help from colleagues as necessary (Moulton et al. 2007), both of which were regularly discussed as effective SRL behaviours in the debriefs with junior doctors. 
Whilst the measurable change in SRL processes was not significant in this study, goal setting and self-monitoring improved among junior doctors that received the intervention with medium effect size $(d=0.53)$ whilst it worsened among junior doctors that did not receive the intervention (see Table 3). Goal setting and self-monitoring skills can be considered as interdependent during an evolving task. Throughout a task, self-regulated learners self-monitor their performance and make necessary adaptive changes to achieve their goals (Zimmerman 2000). This relationship between the two SRL elements makes them difficult to separate, and perhaps is unnatural to do so, given that self-monitoring drives dynamic change in performance to meet the task goals (Leggett et al. 2019). In previous SRL studies, goal setting/self-monitoring were lacking in other simulated environments (Khaled et al. 2016) and hence were of particular interest in this study.

A key aspect in the design of the learning activity within these environments is the use of appropriate event measures that can identify the use of key SRL processes, such as SRL microanalysis (Cleary and Sandars 2011). Although attempts were made to measure these processes at the start and end of the prescribing task, measurement at defined moments as individuals undertake key skills may be necessary to demonstrate the evidence for improved clinical skills acquisition and retention, compared to a traditional 'before and after' measurement approach (Brydges and Butler 2012). Future studies should incorporate similar methods for identifying SRL processes in real time so learners can receive the necessary individualised feedback in the moment.

\section{Self-efficacy}

This study confirmed the importance of helping novices calibrate their self-efficacy or perceived self-confidence with their developing competence. Participants who received 
SRL-enhanced feedback saw an appropriate increase in their prescribing competence and self-efficacy. Participants who did not receive SRL-enhanced feedback increased their sense of self-efficacy in the absence of gains in prescribing competence (Table 4). A number of studies, including this one, demonstrate that providing no feedback, or feedback without attention to SRL processes (including self-efficacy), is unlikely to improve learning (Reynolds et al. 2016), and may provide individuals with a false sense of security about their development (Brinkman et al. 2015), or lead to negative learning gains (Hattie and Timperley 2007). Helping novices calibrate their confidence with their actual competence is a major challenge for medical and healthcare professions education since there is a growing body of evidence that individuals are not developing this metacognitive capability within existing training programmes (Cleary and Sandars 2011; Mavis 2001; Welch et al. 2018). For prescribing and other complex technical skills such as advanced life support, appropriate calibrations are necessary when junior doctors are working in practice, often alone without supervision, and need to make safe clinical decisions (Hautz et al. 2019).

\section{Conclusion}

SRL-enhanced video feedback is an effective educational intervention for improving junior doctor prescribing competency over a four-month workplace-based placement. Video is effective for starting feedback conversations with junior doctors about SRL processes such as goal setting and self-monitoring, when undertaking difficult or challenging prescribing in simulated clinical encounters. Feedback intended to increase self-efficacy or self-confidence by giving praise and positive comments without key information about other SRL processes, is insufficient for sustaining long-term improvements in prescribing competence. The challenge for medical educators is to 
establish the role of SRL-enhanced video feedback across postgraduate training programmes. Further research is necessary to investigate the effectiveness of SRLenhanced video feedback for facilitating the transferability of skills from simulated to workplace-based settings. 


\section{Acknowledgements}

Thanks to the Leicester Kidney Patient Association for supporting and participating in this study. We also thank the doctors who participated in the research and senior clinicians and managers who supported this research.

\section{Declaration of Interest}

This work was funded by Health Education England East Midlands grant number LEI0085. The authors report no declarations of interest. 


\section{References}

Archer JC. 2010. State of the science in health professional education: effective feedback. Med Educ. 44(1):101-108.

Artino Jr AR, Hemmer PA, Durning SJ. 2011. Using self-regulated learning theory to understand the beliefs, emotions, and behaviors of struggling medical students. Acad Med. 86(10):S35-S38.

Bates DW, Cullen DJ, Laird N, Petersen LA, Small SD, Servi D, Laffel G, Sweitzer BJ, Shea BF, Hallisey R, Vander Vliet M. 1995. Incidence of adverse drug events and potential adverse drug events: implications for prevention. Jama. 274(1):29-34.

Bertels J, Almoudaris AM, Cortoos P-J, Jacklin A, Franklin BD. 2013. Feedback on prescribing errors to junior doctors: exploring views, problems and preferred methods. Int J Clin Pharm. 35(3):332-338.

Bing-You R, Varaklis K, Hayes V, Trowbridge R, Kemp H, McKelvy D. 2018. The feedback tango: An integrative review and analysis of the content of the teacherlearner feedback exchange. Academic Medicine. 93(4):657-663.

Bobb A, Gleason K, Husch M, Feinglass J, Yarnold PR, Noskin GA. 2004. The epidemiology of prescribing errors: the potential impact of computerized prescriber order entry. Arch Intern Med. 164(7):785-792.

Boud D, Molloy E. 2013. Rethinking models of feedback for learning: the challenge of design. Assess Eval High Edu. 38(6):698-712.

Bowen L, Marshall M, Murdoch-Eaton D. 2017. Medical student perceptions of feedback and feedback behaviors within the context of the "educational alliance". Acad Med. 92(9):1303-1312.

Brennan N, Mattick K. 2013. A systematic review of educational interventions to change behaviour of prescribers in hospital settings, with a particular emphasis on new prescribers. Br J Clin Pharmacol. 75(2):359-372.

Brinkman DJ, Tichelaar J, van Agtmael MA, de Vries TP, Richir MC. 2015.

Self-reported confidence in prescribing skills correlates poorly with assessed competence in fourth-year medical students. J Clin Pharm. 55(7):825-830.

Brydges R, Butler D. 2012. A reflective analysis of medical education research on self-regulation in learning and practice. Med Educ. 46(1):71-79.

Caldwell G. 2011. Whatever happened to apprenticeship learning? Clin Teach. $8(4): 272-5$. 
Charlton R. 2007. Learning to consult. Oxford (UK): Radcliffe Publishing.

Cleary TJ, Sandars J. 2011. Assessing self-regulatory processes during clinical skill performance: a pilot study. Med Teach. 33(7):e368-e374.

Cohen J. 1992. Statistical power analysis. Curr Dir Psychol Sci. 1(3):98-101.

Daniel M, Rencic J, Durning SJ, Holmboe E, Santen SA, Lang V, Ratcliffe T, Gordon D, Heist B, Lubarsky S, Estrada CA. 2019. Clinical reasoning assessment methods: a scoping review and practical guidance. Acad Med. 94(6):902-912.

Dean B, Schachter M, Vincent C, Barber N. 2002. Causes of prescribing errors in hospital inpatients: a prospective study. The Lancet. 359(9315):1373-1378.

de Bruin AB, Dunlosky J, Cavalcanti RB. 2017. Monitoring and regulation of learning in medical education: the need for predictive cues. Med Educ. 51(6):575-584.

de Vries TPG, Henning RH, Hogerzeil HV, Fresle DA, Policy M, World Health Organization. 1994. Guide to good prescribing: a practical manual (No. WHO/DAP/94.11). Geneva: WHO.

Donisi V, Sibani M, Carrara E, Del Piccolo L, Rimondini M, Mazzaferri F, Bovo C, Tacconelli E. 2019. Emotional, cognitive and social factors of antimicrobial prescribing: can antimicrobial stewardship intervention be effective without addressing psycho-social factors? J Antimicrob Chemother. 74(10):2844-2847.

Dornan T, Ashcroft D, Heathfield H, Lewis P, Miles J, Taylor D, Tully M, Wass V. 2009. An in-depth investigation into causes of prescribing errors by foundation trainees in relation to their medical education. EQUIP study. London: General Medical Council; Dec:1-215.

Duffy MC, Azevedo R, Sun NZ, Griscom SE, Stead V, Crelinsten L, Wiseman J, Maniatis T, Lachapelle K. 2015. Team regulation in a simulated medical emergency: an in-depth analysis of cognitive, metacognitive, and affective processes. Instr Sci. 43(3):401-426.

Dunphy BC, Cantwell R, Bourke S, Fleming M, Smith B, Joseph KS, Dunphy SL. 2010. Cognitive elements in clinical decision-making: toward a cognitive model for medical education and understanding clinical reasoning. Adv Health Sci Educ Theory Pract. 15(2):229-250.

Durning SJ, Cleary TJ, Sandars J, Hemmer P, Kokotailo P, Artino AR. 2011. Perspective: viewing "strugglers" through a different lens: how a self-regulated 
learning perspective can help medical educators with assessment and remediation. Acad Med. 86(4):488-495.

East Midlands Local Education and Training Board (EMLETB). 2017. Postgraduate medical education in the East Midlands. Health Education England. 2017-04-25; [accessed 2020 February 8]. https://www.eastmidlandsdeanery.nhs.uk. Archived by WebCite ${ }^{\circledR}$ at http://www.webcitation.org/6pzUa5oBY.

Farquharson AL, Cresswell AC, Beard JD, Chan P. 2013. Randomized trial of the effect of video feedback on the acquisition of surgical skills. Brit J Surg. 100(11):14481453.

Franklin BD, Reynolds M, Shebl NA, Burnett S, Jacklin A. 2011. Prescribing errors in hospital inpatients: a three-centre study of their prevalence, types and causes. Postgrad Med J. 87(1033):739-745.

General Medical Council (GMC). 2013. Good practice in prescribing and managing medicines and devices. London: GMC; [accessed 2020 February 8]. https://www.gmc-uk.org/ethical-guidance/ethical-guidance-for-doctors/prescribingand-managing-medicines-and-devices.

Hachambachari Y, Fahkarzadeh L, Shariati AA. 2017. The comparison of the effect of two different teaching methods of role-playing and video feedback on learning Cardiopulmonary Resuscitation (CPR). Middle East J Fam Med. 15(8).

Hattie J, Timperley H. 2007. The power of feedback. Rev Educ Res. 77(1):81-112. Hautz WE, Schubert S, Schauber SK, Kunina-Habenicht O, Hautz SC, Kämmer JE, Eva KW. 2019. Accuracy of self-monitoring: does experience, ability or case difficulty matter? Med Educ. 53(7):735-744.

Heikkilä A, Lonka K. 2006. Studying in higher education: students' approaches to learning, self-regulation, and cognitive strategies. Stud High Educ. 31(1):99-117.

Illing JC, Morrow GM, Rothwell nee Kergon CR, Burford BC, Baldauf BK, Davies CL, Peile EB, Spencer JA, Johnson N, Allen M, Morrison J. 2013. Perceptions of UK medical graduates' preparedness for practice: a multi-centre qualitative study reflecting the importance of learning on the job. BMC Med Educ. 13(34).

Kamarudin G, Penm J, Chaar B, Moles R. 2013. Educational interventions to improve prescribing competency: a systematic review. BMJ Open. 3(8):e003291. 
Khaled A, Gulikers J, Biemans H, Mulder M. 2016. Occurrences and quality of teacher and student strategies for self-regulated learning in hands-on simulations. Stud Contin Educ. 38(1):101-121.

Kirschenbaum DS. 1984. Self-regulation and sport psychology: nurturing an emerging symbiosis. J Sport Psychol. 6(2):159-183.

Kuiper RA, Pesut DJ. 2004. Promoting cognitive and metacognitive reflective reasoning skills in nursing practice: self-regulated learning theory. J Adv Nurs. 45(4):381-391.

LaDonna KA, Hatala R, Lingard L, Voyer S, Watling C. 2017. Staging a performance: learners' perceptions about direct observation during residency. Medical education. $51(5): 498-510$.

Larose G, Levy A, Bailey B, Cummins-McManus B, Lebel D, Gravel J. 2017. Decreasing prescribing errors during pediatric emergencies: a randomized simulation trial. Pediatr. 139(3):e20163200.

Leggett H, Sandars J, Roberts T. 2019. Twelve tips on how to provide self-regulated learning (SRL) enhanced feedback on clinical performance. Med Teach. 41(2):147151.

Lindon-Morris E, Laidlaw A. 2014. Anxiety and self-awareness in video feedback. The clinical teacher. 11(3):174-178.

Mavis B. 2001. Self-efficacy and OSCE performance among second year medical students. Adv Health Sci Educ. 6(2):93-102.

McHugh ML. 2012. Interrater reliability: the kappa statistic. Biochem Med. 22(3):276282.

McLellan L, Dornan T, Newton P, Williams SD, Lewis P, Steinke D, Tully MP. 2016. Pharmacist-led feedback workshops increase appropriate prescribing of antimicrobials. J Antimicrob Chemother. 71(5):1415-1425.

Middlemas S, Harwood C. 2018. No place to hide: Football players' and coaches' perceptions of the psychological factors influencing video feedback. Journal of Applied Sport Psychology. 30(1):23-44.

Morgan J, Green V, Blair J. 2017. Using simulation to prepare for clinical practice. Clin Teach. 15(1):57-61. 
Morrow G, Johnson N, Burford B, Rothwell C, Spencer J, Peile E, Davies C, Allen M, Baldauf B, Morrison J, Illing J. 2012. Preparedness for practice: the perceptions of medical graduates and clinical teams. Med Teach. 34(2):123-135.

Moulton CA, Regehr G, Mylopoulos M, MacRae H. 2007. Slowing down when you should: a new model of expert judgment. Acad Med. 82(10 Suppl):S109-S116.

Naik ND, Abbott EF, Gas BL, Murphy BL, Farley DR, Cook DA. 2018. Personalized video feedback improves suturing skills of incoming general surgery trainees. Surgery. 163(4):921-926.

Nesbitt CI, Phillips AW, Searle RF, Stansby G. 2015. Randomized trial to assess the effect of supervised and unsupervised video feedback on teaching practical skills. J Surg Educ. 72(4):697-703.

Nilsen S, Baerheim A. 2005. Feedback on video recorded consultations in medical teaching: why students loathe and love it-a focus-group based qualitative study. BMC medical education. 5(1):28.

Parker H, Farrell O, Bethune R, Hodgetts A, Mattick K. 2019. Pharmacist-led, video-stimulated feedback to reduce prescribing errors in doctors-in-training: a mixed methods evaluation. Br J Clin Pharmacol. 85(10):2405-2413.

Patel R, Green W, Martinez MM, Shahzad MW, Larkin C. 2015. A study of Foundation Year doctors' prescribing in patients with kidney disease at a UK renal unit: a comparison with other prescribers regarding the frequency and type of errors. Eur J Hosp Pharm. 22(5):291-297.

Phillips AW, Matthan J, Bookless LR, Whitehead IJ, Madhavan A, Rodham P, Porter AL, Nesbitt CI, Stansby G. 2017. Individualised expert feedback is not essential for improving basic clinical skills performance in novice learners: a randomized trial. J Surg Educ. 74(4):612-620.

Reynolds M, Jheeta S, Benn J, Sanghera I, Jacklin A, Ingle D, Franklin BD. 2016. Improving feedback on junior doctors' prescribing errors: mixed-methods evaluation of a quality improvement project. BMJ Qual Saf. 26(3):240-247.

Ross S, Loke YK. 2009. Do educational interventions improve prescribing by medical students and junior doctors? A systematic review. Br J Clin Pharmacol. 67(6):662670.

Royal College of Physicians (RCP). 2012. Ward rounds in medicine principles for best practice. London: RCP. 
Royal College of Physicians (RCP). 2015. Generic medical record keeping standards. London: Health Informatics Unit of the RCP. First published in 2007 in Clin Med. 7(4):328-331.

Schunk DH. 2008. Metacognition, self-regulation, and self-regulated learning: research recommendations. Educ Psychol Rev. 20(4):463-467.

Sørensen JL, Østergaard D, LeBlanc V, Ottesen B, Konge L, Dieckmann P, Van der Vleuten C. 2017. Design of simulation-based medical education and advantages and disadvantages of in situ simulation versus off-site simulation. BMC medical education. 17(1):20.

Sullivan GM, Feinn R. 2012. Using effect size-or why the $P$ value is not enough. J Grad Med Educ. 4(3):279-282.

Swinscow TDV, Campbell MJ. 1997. Study design and choosing a statistical test. Statistics at Square One. 9(13). London: BMJ.

Tanner KD. 2012. Promoting student metacognition. CBE-Life Sci Educ. 11(2):113120.

Wall D, Bolshaw A, Carolan J. 2006. From undergraduate medical education to preregistration house officer: how prepared are students? Med Teach. 28:435-439.

Welch P, Young L, Johnson P, Lindsay D. 2018. Metacognitive awareness and the link with undergraduate examination performance and clinical reasoning. Med Ed Publish. 7(2):32.

Zimmerman BJ. 1989. A social cognitive view of self-regulated academic learning. J Educ Psychol. 81(3):329.

Zimmerman BJ. 1990. Self-regulated learning and academic achievement: an overview. Educ Psychol. 25(1):3-17.

Zimmerman BJ. 2000. Self-efficacy: an essential motive to learn. Contemp Educ Psychol. 25(1):82-91. 


\section{Practice points}

1. Medical school graduates in the UK have reported feeling underprepared for the task of prescribing when embarking on practice.

2. Effective application of self-regulated learning (SRL) has been demonstrated as an approach to improve performance on practice-based tasks through selfreflection.

3. Findings from this study demonstrate for the first time that SRL-enhanced video feedback following simulated clinical encounters is effective in improving medicines prescribing competency and the development of the SRL processes of goal setting and self-monitoring skills required for safe and effective medicines prescribing.

4. SRL-enhanced video feedback should be used more widely in the training and development of junior doctors. 
Control cohort

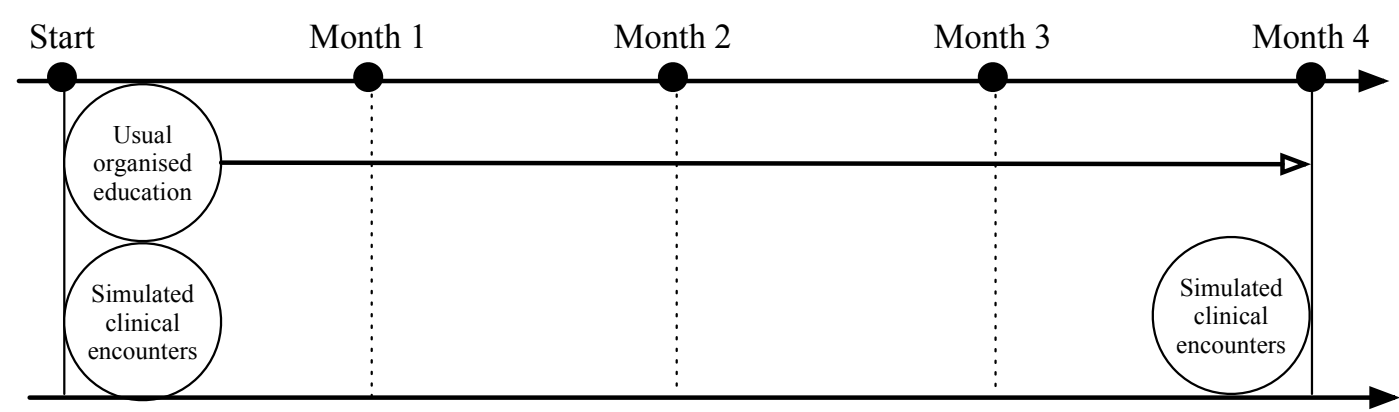

Intervention cohort

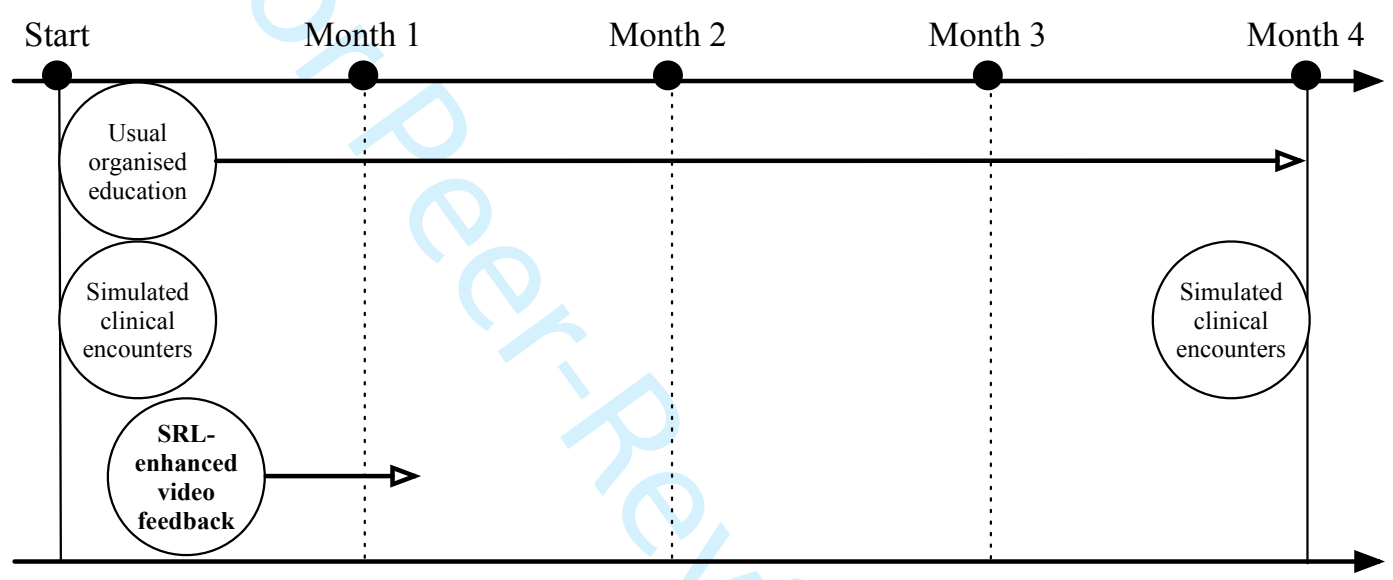

Figure 1. Control (upper) and intervention (lower) timelines. The intervention cohort received SRL-enhanced video feedback at their request when it could fit with the junior doctors' work schedule. 
Table 1. SRL questionnaire used to capture SRL score.

\begin{tabular}{l|c} 
Question & Response type \\
\hline 1) How confident are you feeling about completing the ward round, on a scale & Scale 0-100 \\
of 0-100? & Free text \\
\hline 2) What do you use to judge your confidence? & Free text \\
\hline 3) What are you thinking about as you prepare for the ward round? & Free text \\
\hline 4) What goals do you have in mind? & Free text \\
\hline 5) What do you need to do to successfully complete the ward round? & Free text \\
\hline 6) Do you have a particular technique you will follow? & Scale 0-100 \\
\hline 7) How satisfied are you feeling with your ward round on a scale of 0-100? & Free text \\
\hline 8) What did you use to judge your satisfaction? & Scale 0-100 \\
\hline 9) If you were to manage this task again, what might you do the same or \\
differently? & Free text \\
\hline 10) How sure are you on a scale of 0-100 that you could successfully complete & another simulated clinical encounter in the future?
\end{tabular}


Table 2. Demographics of participants for both control and intervention cohorts - all participants participated in two sets of simulated clinical encounters.

\begin{tabular}{|c|c|c|c|c|c|c|c|c|}
\hline \multirow{2}{*}{ Occupational grade } & \multirow{2}{*}{$\begin{array}{l}\text { Years post- } \\
\text { graduation }\end{array}$} & \multicolumn{3}{|c|}{ Cohort 1 - control } & \multicolumn{3}{|c|}{ Cohort 2 - intervention } & \multirow{2}{*}{ Total } \\
\hline & & Female & Male & Total & Female & Male & Total & \\
\hline Foundation Year 1 & 0 & 2 & 2 & 4 & 2 & 3 & 5 & 9 \\
\hline Foundation Year 2 & 1 & 1 & & 1 & 2 & 2 & 4 & 5 \\
\hline Core Trainee Year 1 & $\geq 2$ & & & & & 2 & 2 & 2 \\
\hline Core Trainee Year 2 & $\geq 3$ & & & & 1 & & 1 & 1 \\
\hline $\begin{array}{c}\text { Senior House } \\
\text { Officer }\end{array}$ & $\geq 2$ & & 1 & 1 & & & & 1 \\
\hline Total & & 3 & 3 & 6 & 5 & 7 & 12 & 18 \\
\hline
\end{tabular}


Table 3. Pre- and post-intervention data for prescribing competency and SRL scores for both cohorts.

\begin{tabular}{|c|c|c|c|c|c|c|c|c|}
\hline \multirow{3}{*}{ Variable } & \multicolumn{4}{|c|}{ Cohort 1 - control } & \multicolumn{4}{|c|}{ Cohort 2 - intervention } \\
\hline & \multicolumn{2}{|c|}{ Central tendency } & \multirow{2}{*}{$\begin{array}{l}\text { Average } \\
\text { change }\end{array}$} & \multirow[t]{2}{*}{$P$ value } & \multicolumn{2}{|c|}{ Central tendency } & \multirow{2}{*}{$\begin{array}{l}\text { Average } \\
\text { change }\end{array}$} & \multirow[t]{2}{*}{$\mathrm{P}$ value } \\
\hline & $\begin{array}{c}\text { Pre- } \\
\text { intervention }\end{array}$ & $\begin{array}{c}\text { Post- } \\
\text { intervention }\end{array}$ & & & $\begin{array}{c}\text { Pre- } \\
\text { intervention }\end{array}$ & $\begin{array}{c}\text { Post- } \\
\text { intervention }\end{array}$ & & \\
\hline $\begin{array}{l}\text { Prescribing } \\
\text { competency }\end{array}$ & 0.448 & 0.493 & $\begin{array}{c}0.0446 \\
(-0.132- \\
0.043)\end{array}$ & 0.246 & 0.397 & 0.545 & $\begin{array}{c}0.148 \\
(0.082- \\
0.215)\end{array}$ & $<0.001 * * *$ \\
\hline Total SRL score & 14.98 & 14.58 & $\begin{array}{c}-0.04 \\
(-2.64- \\
3.44)\end{array}$ & 0.749 & 13.82 & 14.71 & $\begin{array}{c}0.883 \\
(-0.953- \\
2.712)\end{array}$ & 0.312 \\
\hline Self-efficacy & 1.100 & 1.400 & $\begin{array}{c}0.40 \\
(0.523- \\
1.410)\end{array}$ & $0.026 * *$ & 1.067 & 1.200 & $\begin{array}{c}0.133 \\
(-0.021- \\
0.287)\end{array}$ & $0.083^{*}$ \\
\hline $\begin{array}{l}\text { Goal setting and } \\
\text { self-monitoring }\end{array}$ & 7.800 & 6.817 & $\begin{array}{l}-0.083 \\
(1.150- \\
1.583)\end{array}$ & 0.246 & 6.000 & 7.37 & $\begin{array}{c}1.367 \\
(-0.288- \\
3.022)\end{array}$ & $0.096 *$ \\
\hline
\end{tabular}

\#Where central tendency is either mean or median, for normal and non-normal data, respectively.

$*=$ Significant at $\mathrm{p}<0.10 ; * *=$ Significant at $\mathrm{p}<0.05 ; * * *=$ Significant at $\mathrm{p}<0.01$. 
Table 4. Intervention cohort correlations for SRL subprocesses goal setting and selfmonitoring skills compared to competency scores. Simulation 1 and 2 scores relate to scores in simulations conducted at the beginning (Simulation 1) and end (Simulation 2) of the four-month rotation.

\begin{tabular}{l|l|c|c|}
\hline \multicolumn{2}{|c|}{ Intervention cohort (n=12) } & \multicolumn{2}{c|}{ Prescribing competency scores } \\
\cline { 3 - 4 } & & Simulation 1 & Simulation 2 \\
\hline Self-efficacy & Simulation 1 & 0.192 & 0.391 \\
\cline { 2 - 4 } & Simulation 2 & 0.487 & $0.591^{*}$ \\
\hline \multirow{2}{*}{$\begin{array}{l}\text { Goal setting and } \\
\text { self-monitoring }\end{array}$} & Simulation 1 & -0.149 & 0.046 \\
\hline
\end{tabular}

$*=$ Significant at $\mathrm{p}<0.05$. 
Table 5. Example quotes from the participant debrief interviews.

\section{Theme: Perceptions of simulations}

It's very good to have real patients because...er... with the dummies that we used in previous simulations...there are a lot of limitations...um...with real patients, it just helps to make the whole simulation seem a lot more real...er...you take it a lot more seriously...erm...and...um... in terms of patients talking to you and you're trying to figure out what's going on at the same time... it's a very real distraction that you don't have with the dummies... So, yes, it was good [Control cohort, JD01].

I think...in all honesty, I think having two sim's is quite good because...especially the one at the beginning because starting in renal you're not really sure what's what's going on...even though it's a third F1 job...it's...it's kind of you're thrown in the deep end no matter what...so I think the sims then was a good idea to see what...the kind of... sort of the meat, the bread and butter of normal renal is. I think having another sim shows... how much...you've think you've probably increased in confidence... how you're able to conduct a ward round...things that would probably be a bit worrying and that I wouldn't be sure of...back then... are a bit more clear now...[Control cohort, JD02].

so, it's a really good reason for getting off the wards for half a day, and yeah, it's a good learning tool...I think it could be quite intimidating if you're coming straight from being a medical student as an F1 doctor...I think I would find that very, very hard, but I think you just have to remember you are not being assessed... it's just...it's fun as well...to just see it that way, you know, and it's just... it's a good experience. [Intervention cohort, JD05].

...it was an implemented torture...The first one I didn't enjoy that much...but I think it was because I'd only been a doctor for 2 weeks...the second session was a lot better...erm...well...I probably would [encourage a friend to participate...I...I think... doing the handover to someone...realizing that actually...I can handover...I mean that's quite useful [Intervention cohort, JD08].

overall...it's very positive. I think I've learned a lot first time round... although I've come out of this one going I'm knackered...that was quite stressful and quite hard work...I'm sure the same will be true this time to be honest... [Intervention cohort, JD11]

\section{Theme: Perception towards feedback}

Absolutely, I would have no reservations recommending them to anyone...even at the beginning I was unsure, because I...there is lots of things about being filmed and going round and doing things that I don't like...it's not my kind of thing, but I find it so valuable and I've got things from it that I didn't expect to...that I would suggest to anyone unless 
that they think they're the best doctor in the world...that there is something they can learn by doing this... and in different setting I think it could be used really really well as well...Intervention cohort, JD03].

I think there is much more to gain from the feedback... from the exercise itself after having feedback, rather than going away...it gives you a much better sort of....all-round picture and it gives you goals also to focus on... So I would say it's valuable...Intervention cohort, JD04]

[watching yourself on film is a] little bit cringe worthy, to be honest, watching myself on film, I don't enjoy doing that, but...erm...they make you...they make you learn...yeah, I still remember the cases quite vividly and the details quite vividly, so I think it was really beneficial. [Intervention cohort, JD05]

[The simulations and feedback] help you to then go back on to the wards and...develop a more systematic approach to your... ward round as it were...I enjoyed the first ones, they really went quite well...I was more nervous because I didn't know what to expect... but I think on the whole I was pleased... The feedback, identified some, you know...fairly major things that you need to think about that I hadn't given enough thought to...so erm...yeah...it was definitely worth doing from that perspective... The second one, I think was really just to kind of...a good measure of how much you'd improved from the first one... and...I felt a lot more confident going into it... and...I knew what information I wanted from each patient... and...you know...more so than I did in the first one....and this sort of helped me structure my approach. I was far more interested...the patients had already been clerked...so I was far more interested in seeing what result we already had... and...letting that guide what I did next with the patient... whereas before I went straight into the patient...in the first one...took a history and examined them, and then I've looked at what had been done... and so I approached it in a much more practical manner this time... and that made me quicker, and I think probably more effective...I couldn't have done that the first time just because you didn't know what you're expecting... and I felt the second one...yeah...I think like...even (?) went better... and... but then it's nice to... because we're in a such an early stage in our career, it's still nice to be able to identify some decent...you know...learning points from that second one as well...that you still have got outstanding from the first one...so...yeah...no...on the whole I felt they went really well, I've really enjoyed it... [Intervention cohort, JD10] 